\title{
Polarisierte Städte: Die AfD im urbanen Kontext. Eine Analyse von Wahl- und Sozialdaten in sechzehn deutschen Städten
}

\author{
Jan Lucas Geilen ${ }^{1}$ and Daniel Mullis ${ }^{2}$ \\ ${ }^{1}$ Fachbereich Gesellschaftswissenschaften und Philosophie, Institut für Politikwissenschaft, \\ Philipps-Universität Marburg, 35032 Marburg, Germany \\ ${ }^{2}$ Leibniz-Institut Hessische Stiftung Friedens- und Konfliktforschung (HSFK), \\ 60329 Frankfurt am Main, Germany \\ Correspondence: Daniel Mullis (mullis@hsfk.de)
}

Received: 8 July 2020 - Revised: 21 December 2020 - Accepted: 4 March 2021 - Published: 15 April 2021

\begin{abstract}
Kurzfassung. For the first time since 1945, with the Alternative für Deutschland (AfD) a far-right party has now consolidated itself in Germany on all political scales. In the political sciences as well as sociology, but not so much in human geography, there is much debate on the funding reasons. The debate is polarized and unfolds between two positions: on the one hand economic factors are marked as principal forces, on the other a cultural backlash. Electoral analyses have focused on the spatial division of the AfD's success between urban and rural areas as well as West and East Germany. By contrast, we focus on urban divisions. We examine the AfD's results in the general election of 2017 on the scale of districts in the largest city of each federal state by analyzing the correlation of the share of AfD votes with social data. In contrast to the polarized debate in the social sciences we choose a multidimensional perspective and analyze social data related to class, migration, acceptance of democracy and age. First, the resulting picture is heterogeneous across the cities and does not show a pronounced East-West polarization. Second, economic factors as well as factors related to migration correlate with the share of the AfD, whereby the former are pronounced somewhat stronger. Third, lack of trust in democracy is the most uniform indicator for a strong showing of the AfD in our sample; and fourth, in all cities there are districts which run counter to the generalized picture. We can show that in urban settings it is primarily, but not exclusively, in marginalized districts that the AfD has its strongholds. But this does not mean that all marginalized districts are dominated by the far-right party. In order to better understand the spatially highly fragmented social processes, in particular more qualitative research is needed.
\end{abstract}

\section{Einleitung}

Extrem rechte und autoritäre Politiken erstarken weltweit. In Deutschland steht hierfür nicht zuletzt der Aufstieg der $\mathrm{Al}$ ternative für Deutschland (AfD). Scheiterte die Partei 2013 noch am Einzug in den Bundestag, wurde sie 2017 mit $12,6 \%$ drittstärkste Kraft. Nach weiteren Erfolgen auf Ebene der Länder ist mit der AfD seit 2018 zum ersten Mal seit 1945 in Deutschland eine Rechtsaußen-Partei zugleich im europäischen Parlament, im Bundestag sowie in allen Landesparlamenten vertreten. Seit ihrer Gründung 2013 hat sie sich sukzessive nach rechts außen bewegt und spätestens seit
2015 bilden ,,autoritäre, völkisch-nationalistische, homophobe, antifeministische, antisemitische und geschichtsrevisionistische Positionen“ die zentralen Bezugspunkte (Celik et al., 2020: 150). Der Blick auf ihre Basis verdeutlicht hingegen, wie sehr die Partei seit Anbeginn im rechtsextremen Milieu verankert war (Celik et al., 2020: 172). Trotz dieser politischen und gesellschaftlichen Verschiebungen sind in der Humangeographie (Lizotte, 2018) und insbesondere in der deutschsprachigen die Auseinandersetzungen mit der extremen Rechten lange Zeit marginal geblieben (Bürk, 2012: 28) - dies ändert sich gerade. 
Jüngst sind diverse Beiträge zum Thema erschienen (vgl. Belina, 2020; Hövel, 2018; Klüter, 2020; Mullis, 2021; Oßenbrügge, 2018), die sub \urban hat ein Themenheft veröffentlicht (Bernet et al., 2019), und auch beim Deutschen Kongress für Geographie 2017 und insbesondere 2019 wurden rechts-autoritäre Politiken diskutiert. In den Politikwissenschaften sowie der Soziologie, wo intensiver über die Verschiebungen debattiert wird, besteht weitestgehend Einigkeit: Der Erfolg der extremen Rechten ist „Ergebnis eines Zusammenwirkens von Globalisierungs- und Neoliberalisierungskrisen" (Geiselberger, 2017: 12). Gestritten wird hingegen darüber, ob eher sozio-ökonomische oder identitätspolitische Faktoren ausschlaggebend sind. Es finden sich aber auch Stimmen, die dafür plädieren, die Ursachen multidimensional zu betrachten (vgl. Mullis und Zschocke, 2019: 22-29) - wir werden uns auf letztere Position beziehen.

Im Folgenden gehen wir der Frage nach, was die Ursachen des Erfolgs der AfD in Städten sind bzw. in welchen städtischen sozialen Gefügen sie diesen feiert. Ausgangspunkt unserer Fokussierung auf Stadt sind zwei Befunde: Erstens, die AfD feiert in (Groß-)Städten in Ost- und Westdeutschland Erfolge. So kamen etwa in Hessen immerhin rund ein Drittel der knapp 400000 Zweitstimmen für die AfD bei der Bundestagswahl 2017 aus urbanen Ballungszentren (Belina, 2019); und mit Dresden und Leipzig finden sich zwei Metropolen, in denen die AfD besser als auf Bundesebene abschnitt (Bernet et al., 2019: 13). Zweitens, der Zuspruch zur AfD innerhalb der Städte ist stark polarisiert. Beide Aspekte wurden bisher kaum diskutiert. Indem wir die Diskussion aufgreifen, zeigen wir, dass die Fokussierung auf die städtischen Gesamtergebnisse zu kurz greift und Städte vorschnell als kosmopolite Orte abseits von rechten Raumnahmen verklärt werden. Wir ergänzen hiermit die bisherigen Analysen, die vor allem die Stadt-Land- sowie West-Ost-Polarisierung herausgestellt haben (Kapitel 3). Leitend für die Untersuchung sind die Debatten und Befunde zum Erstarken der AfD (Kapitel 2). Von diesen ausgehend untersuchen wir mittels einer bivariaten linearen Regression (Kapitel 4), inwiefern der Zweitstimmenanteil der AfD mit Indikatoren für sozio-ökonomische Lage, Migration, Zuspruch zur Demokratie und Altersstruktur in Städten korreliert (Kapitel 5). Leitend ist die These, dass in sozio-ökonomisch marginalisierten Stadtteilen die AfD eher gewählt wird als in anderen. Betrachtet haben wir die jeweils größte Stadt je Bundesland. Die Auswahl ist nicht repräsentativ, gibt aber einen guten Eindruck über die Situation im gesamten Bundesgebiet und eröffnet Anschlussmöglichkeiten für weitere Untersuchungen.

Um möglichst einheitliche Daten über alle Städte hinweg zu haben, wurden die Wahlergebnisse der Bundestagswahl herangeozogen. Referenzjahr für die Sozialdaten ist 2017. Da es keinen bundesweiten stadtteilbezogenen Datensatz gibt, barg dessen Zusammenstellung einige Herausforderungen. Dies vor allem, weil in den Kommunen Indikatoren nicht einheitlich erfasst sind. Ein statistischer Vergleich zwischen den Städten ist deshalb nicht möglich. Aussagen lassen sich nur für die Zusammenhänge innerhalb der Städte treffen. Insgesamt hat sich - in Anbetracht des Forschungsstandes nicht ganz unerwartet - ein heterogenes Bild ergeben. Weder die sozio-ökonomische Lage noch die Anwesenheit von Migrant*innen weisen durchweg einen Zusammenhang mit dem Zweitstimmenanteil der AfD auf. Relativ stabil und durchgehend wirkt sich jedoch eine niedrige Wahlbeteiligung positiv auf die Zweitstimmen der AfD aus. Gleiches gilt für den Anteil an Jugendlichen, wenn auch in umgekehrter Weise. Auch erkennen wir in den Ergebnissen kein deutliches Ost-West-Muster. In allen Städten finden wir Stadtteile, die so gar nicht dem statistischen Bild entsprechen. Wir sind der Meinung, dass über diese nicht einfach hinweggegangen werden kann und die Analyse des Spannungsverhältnisses aus beschreibbarer Korrelation und Diskontinuität die gesellschaftlichen Entwicklungen klarer zu fassen erlaubt.

\section{Ursachen des Erstarkens der AfD}

In der Einstellungsforschung wird für Deutschland auf zwei gegenläufige Tendenzen verwiesen: Einerseits wird die Gesellschaft liberaler und extrem rechte Einstellungen nehmen $\mathrm{ab}$; andererseits nimmt die Polarisierung zu und die extreme Rechte vollzieht einen Radikalisierungsprozess. Auch sind autoritäre Aggression und Unterwürfigkeit sowie mitunter klar antisemitische Verschwörungsmentalitäten verbreitet. Dabei untergraben diese autoritären Dynamiken demokratische Normen auch jenseits klassischer rechtsextremer Einstellungen (Decker et al., 2020). Für Matthias Quent (2019: 55) handelt es sich bei den Bestrebungen von Rechtsaußen um „einen erbitterten Versuch“, die liberalen Terraingewinne zurückzudrängen. So seien in den letzten Jahren die Menschen nicht ,massenhaft [...] rechtsradikal geworden“, eher sei das Potenzial „zum Handeln aktiviert“ worden (Quent, 2019: 48). Rechtsextreme Einstellungen, allen voran rassistische Ressentiments und antidemokratische Haltungen, waren lange vor dem Aufstieg der AfD in der sogenannten Mitte der Gesellschaft verankert. Parallel zum Erstarken der AfD und der anhaltenden Debatte über Migration und Flucht nahmen „manifest ausländerfeindliche“ Einstellungen nach Jahren des Rückgangs zwischen 2014 und 2018 deutlich auf $24,1 \% \mathrm{zu}$; sanken 2020 jedoch wieder auf den insgesamt niedrigsten je gemessenen Wert von 16,5\% (Ost: 27,8\%/West: 13,7\%) (Decker et al., 2020: 49). Anhaltend hoch - im Langzeittrend jedoch rückläufig - ist die Wahrnehmung von politischer Ohnmacht: 72,9\% (Ost: 83,6\%/West: $70,2 \%$ ) sind der Meinung, „Leute wie ich haben sowieso keinen Einfluss darauf, was die Regierung tut" (Decker et al., 2020: 69). Mit der „Demokratie, wie sie in der Bundesrepublik funktioniert", sind gerade mal 57,6\% zufrieden (Ost: 40,7\%/West: 61,8\%) (Decker et al., 2020: 62).

Mit der AfD ist eine politische Kraft entstanden, die zum einen Menschen, die sich von der Demokratie enttäuscht und den Eliten ungehört fühlen, politisch reintegriert (Heit- 
meyer, 2018: 212) sowie zum anderen das in der Gesellschaft vorhandene autoritäre und extrem rechte Potential an sich bindet (Decker et al., 2020: 74). Die Aktivierung von Nichtwähler*innen ist zentral (Heitmeyer, 2018: 192-194). Die Wahlbeteiligung bei Bundestagswahlen sank seit den 1970ern ziemlich kontinuierlich von 91,1 \% im Jahr 1972 auf $200970,8 \%$, seither steigt sie wieder etwas an auf 2017 insgesamt 76,2\%. Wilhelm Heitmeyer (2018: $188 \mathrm{ff}$.) betont in diesem Zusammenhang, dass dieser Rückgang seitens ,konservativer Sozialwissenschaftler" als wachsende stille $\mathrm{Zu}$ stimmung mit der bestehenden Ordnung gedeutet wird. Eher sei es jedoch so, dass sich die Menschen ,,aufgrund von Unzufriedenheit aus dem demokratischen Prozess“ zurückziehen und damit eine niedrige Wahlbeteiligung als Indikator für eine wachsende (stille) Wut zu betrachten sei. Gewählt wird die AfD vor allem von Männern im Alter zwischen 31 und 60 Jahren (Celik et al., 2020: 158 ff.). Auffällig stark ist sie zudem in Regionen, in denen der Anteil älterer Menschen hoch ist (Franz et al., 2018). Im Langzeittrend werden Konjunkturen deutlich: 2014 sind es nicht zuletzt „Menschen mit höherem Bildungsgrad“" und mit „,vorwiegend mittlerem und höherem Einkommen“, die die Rechtsaußen-Partei wählen. 2016 - und damit auch in der Zeit der Bundestagswahl - nimmt der Anteil an Menschen ,,mit niedrigerem Bildungsgrad" sowie einem geringeren Einkommen zu (Celik et al., 2020, 170; vgl. Lengfeld, 2018). Dieser Trend setzt sich 2020 nicht fort. Das Einkommensniveau der Wähler*innen steigt, sodass heute das Potenzial der AfD verstärkt wieder in der ,gehobenen Mitte der Gesellschaft" liegt (Celik et al., 2020: 170). Zugleich ist die Basis der Partei rechtsextremer geworden (Celik et al., 2020, 166), was darauf hindeutet, dass die Normalisierung solcher Positionen in Teilen der oberen Schichten voranschreitet (vgl. Heitmeyer, 2018, 208).

In der Politikwissenschaft und der Soziologie wird intensiv über die Ursachen des Erstarkens der extremen Rechten diskutiert (vgl. Mullis und Zschocke, 2019). Dabei liegt der Fokus auf Globalisierung und Krisen der Neoliberalisierung (vgl. Geiselberger, 2017). Grundsätzlich bestehen hier Anknüpfungspunkte zu den in der Humangeographie seit den 1980ern geführten Debatten zu Dynamiken der Neoliberalisierung (des Städtischen). Nicht zuletzt wurden hierbei die zunehmende soziale Spaltung sowie Exklusion aus demokratischer Teilhabe betont (vgl. Brenner und Theodore, 2002). Umstritten ist indes, ob eher ökonomische Faktoren wie sozialer Abstieg und Prekarisierung oder Rassismus, Demokratieverdruss sowie kulturelle Fragen stärker ins Gewicht fallen. Die erste Position wird mit unterschiedlicher Absolutheit vorgetragen: Für Oliver Nachtwey (2016) ist es die ökonomische Deprivation, die in einer neuen Abstiegserfahrung kulminiert, die - nicht nur, aber auch - das rechte Aufbegehren begründet. Philipp Manow (2018: 33) deutet den Aufstieg der AfD weniger als Folge von Deprivation denn als (rassistischen) Absicherungskampf von tendenziell noch besser gestellten Facharbeiter*innen, die sich gegen $\mathrm{Zu}$ - wanderung ins Sozialversicherungssystem wehren (Manow, 2018: 99-102). Dies begründet er unter anderem damit, dass mit einem höheren Anteil an sozialversicherungspflichtiger Beschäftigung der Zweitstimmenanteil der AfD steigt (Manow, 2018: 96). Relevant seien auch Erfahrungen von Arbeitslosigkeit in der Erwerbsbiographie, zumal dies nachhaltig das Abstiegsrisiko vor Augen führe (Manow, 2018: 99). Prozesse der gesellschaftlichen Desintegration sind sodann für Heitmeyer (2018: 344) ursächlich. Diese würden primär aus dem Zusammenspiel von Wettbewerbsdruck des globalisierten ,,autoritären Kapitalismus“, sozialer Vereinzelung und der sukzessiven Entleerung demokratischer Teilhabe erwachsen (Heitmeyer, 2018: 197).

Diese Ansätze kritisieren Gurminder Bhambra (2017) wie auch Emma Dowling et al. (2017: 411) dafür, dass (zumindest tendenziell) sozio-ökonomische Erklärungen ,zu Lasten anderer", die auf Ungleichwertigkeitsideologien abzielen, priorisiert werden. Konflikte um Identität und Kultur, um Geschlechterrollen sowie die Wirkmächtigkeit von Rassismus - die zwar mit sozio-ökonomische Fragen zusammenhingen, aber nicht auf diese zu reduzieren seien - würden in ihrer Bedeutung unterschätzt. Diverse Studien zusammenfassend, argumentiert Holger Lengfeld (2018), dass es das „Gefühl der kulturellen Bedrohung durch die Zuwanderung von ethnisch-kulturell fremden Menschen nach Deutschland“ ist, welches für das Erstarken extrem rechter Politiken verantwortlich zeichnet. Diesen Befund stützen Ronald Inglehart und Pippa Norris (2016) in einer europaweit vergleichenden Studie. Sie arbeiten heraus, dass die einenden Merkmale von Wähler*innen von Rechtsaußen-Parteien in der Ablehnung kosmopolitischer, pluralistischer und post-materialistischer Lebensmodelle sowie in einer grundlegenden Skepsis gegenüber Zuwanderung liegen. In diesem Sinne betont auch Quent (2019: 64), dass aktuell eher ein Kultur- als ein Klassenkampf im Gange ist.

Auf der Ebene von Stadtteilen wird über die „Konsequenzen wachsender ethnisch-kultureller Vielfalt für den sozialen Zusammenhalt" (Gundelach, 2017: 216) seit Jahrzehnten gestritten. Im Kern stehen sich die Konflikt- und Kontakttheorie gegenüber (Gundelach, 2017: 210 ff.): Im ersten Fall wird davon ausgegangen, dass mit zunehmender (ethnischer) Diversität in Stadtteilen Vertrauen in Gesellschaft ab- und Konflikte zunehmen und der soziale Zusammenhalt im Zuge von Zuwanderung schwindet. Im zweiten werden Konflikte nicht negiert, es wird aber betont, dass zunehmender Austausch über die Zeit Misstrauen abbaut und Kontakt einen Beitrag zur Minderung von Ressentiments leistet. Die Debatte hält an, zumal beide Thesen empirisch bestätigt wurden (Blokland, 2011; Gundelach, 2017). Beide Positionen sind jedoch nicht unproblematisch, zumal sie die Kategorie Ethnizität essentialisieren. Darüber hinaus finden sie die Ursache für die Konflikte beim Auftreten von als Immigrant*innen identifizierter Menschen, anstelle nach rassistischen Ressentiments zu fragen, die die vermeintlich Anderen erst zu Anderen machen. 
Empirische Befunde unterlaufen diese polarisierte Debatte, indem sie die Vielschichtigkeit der sozialen Prozesse offenlegen. Aufgezeigt wurde etwa, dass in Westdeutschland eher sozio-ökonomische Marginalisierung als Prädiktor für die Wahl der AfD ausschlägt, während im Osten Ressentiments stärker ins Gewicht fallen (Deppisch et al., 2019: 78 ff.). Daniel Mullis und Paul Zschocke (2019: 27-29) argumentieren in ihrer empirisch geleiteten Literaturschau dafür, den Erfolg der extremen Rechten multidimensional zu betrachten. Vier Faktoren stellen sie hierfür zentral: Abstieg, Entdemokratisierung, soziale Lage und Rassismus. Abstiegserfahrungen sowie Gefühle der Zurücksetzung seien erstens von Bedeutung, sollten aber nicht alleine sozio-ökonomisch verstanden werden. Es gehe auch um verletzte Männlichkeit oder die Erfahrung von Bedeutungsverlusten der ,eigenen" nationalen Wirtschaftsmacht. Zweitens würden gerade Erfahrungen des Verlustes von demokratischer Einbindung schwer wiegen und viele betreffen. Drittens sei die soziale Lage von Relevanz, zumal ,die Bürden wie auch die Ressourcen, um mit der Globalisierung von Kapital und Migration, neoliberaler Individualisierung und Marktansprüchen umzugehen, in der Gesellschaft sehr ungleich verteilt" (Mullis und Zschocke, 2019: 28) seien. Und viertens müsse der Faktor Rassismus betrachtet werden, zumal dieser das Fundament dafür bilde, dass Verteilungskonflikte überhaupt rassifiziert in weißen Identitätspolitiken artikuliert würden. Die Dimensionen seien nicht aufeinander reduzierbar und keinem der Faktoren könne ein Primat zur Erklärung der gegenwärtigen Tendenzen zugedacht werden.

\section{Räumliche Repräsentationen}

In den bisher eingeführten Debatten wurden zwei räumliche Polarisierungen hervorgehoben: Zum einen eine Ost-West und zum anderen eine Land-Stadt, wobei die AfD jeweils in Ersterem stärker abschnitt. So erzielte die Partei 2017 in den neuen Bundesländern Spitzenresultate um jeweils ca. $20 \%$ - in Sachsen wurde sie mit $27 \%$ stärkste Kraft -, während sie in den westdeutschen Bundesländern im Schnitt um die $10 \%$ erreichte. Bei den Europawahlen 2019 war das Bild auf Grund von Verlusten der AfD in Westdeutschland noch akzentuierter. Bei den drei Landtagswahlen im Herbst 2019 in Brandenburg, Sachsen und Thüringen konsolidierte die Partei ihre Ergebnisse bei jeweils rund 25\% (Mullis und Zschocke, 2019: 6-12). Im zweiten Fall wird der Erfolg der AfD im ländlichen Raum hervorgehoben. Die AfD feiert ihre Erfolge insbesondere in ,dünn besiedelten Räumen mit Überalterungsproblemen“ (Franz et al., 2018). Es ist die Rede von der „Rache der Dörfer“ (Kaschuba, 2016) gegen die liberalen und offenen Stadtgesellschaften. Larissa Deppisch et al. (2019: 81-84) zeigen hingegen, dass das Bild nicht einheitlich ist: Der Faktor ländliche Region fällt im Osten weit stärker ins Gewicht als im Westen und es sind nicht die „sehr“, sondern die „eher“ ländlichen Regionen, in denen die AfD stark abschneidet.

Zwei Aspekte sind an dieser räumlichen Fokussierung problematisch: Erstens sind beide Interpretationen geprägt von Ressentiments, wonach der Erfolg der AfD auf Rückständigkeit, mangelnder Aufklärung und tradiertem Autoritarismus beruhe (vgl. Förtner et al., 2019; Quent, 2016), womit umgekehrt Städte als kosmopolite Orte mit gesellschaftsliberalem Fundament verklärt werden. Für die besonders wirkmächtige Ost-West-Polarisierung argumentiert Quent (2016: 103-107), dass der Osten des Landes noch immer als „Sonderfall“, als Abweichung vom „Normalfall“ Westdeutschland gilt. Werden jedoch soziale und demografische Faktoren als Indikatoren für das Abschneiden der AfD angelegt, wird offenkundig, dass diese Zuschreibungen nicht tragen (Franz et al., 2018). „Ein genuiner, womöglich sogar kulturell bedingter Ost-Faktor" kann, so betonen Richard Hilmer et al. (2017:27), ,nur in begrenztem Maße“ festgestellt werden. Für Maximilian Förtner et al. (2019: 40) indes ist klar, ,dass ein herkömmliches Verständnis von Stadt und Land im Sinne homogener und stabiler Räume für das Verständnis der Geographie rechtspopulistischer Mobilisierungen zu kurz greift." Sie plädieren stattdessen dafür, auf die ungleichen Dynamiken von Urbanisierung sowie die Herausbildung von Zentralität und Peripherie zu fokussieren. Zweitens ist die Territorialisierung von gesellschaftlichen Konflikten problematisch. Stefan Kipfer und Mustafa Dikeç (2019: 45) betonen, dass die Analyse von ganzen Kommunen letztlich kaschiert, dass verhältnismäßig tiefe Prozentzahlen bei Wahlen in Städten eine weit größere Zahl an Menschen im Umfeld der extremen Rechten bedeuten kann, als hohe in ländlichen Regionen. Gerade für die Bedrohungslage von marginalisierten Gruppen ist dies bedeutsam. Bernd Belina (2017: 201) schließt, dass es keinen Sozialraum gibt, der per se vor rechten Erfolgen schützen würde, noch gebe es den rechten Raum. Die Analyse räumlicher Verteilungen könne jedoch hilfreich sein, um die richtigen Fragen zu stellen. Sie seien aber niemals ein Erklärendes, sondern immer ein zu Erklärendes. Dies werden wir berücksichtigen, wenn wir nun selbst räumliche Polarisierungen des Wahlverhaltens in Städten auswerten.

\section{Vorgehen und Datensatz}

Leitend für die Befragung der Gründe für die Wahl der AfD im städtischen Kontext ist das Plädoyer für eine multidimensionale Analyse. Für Städte gibt es keine einheitlichen Erhebungen zu Einstellungsmustern oder Erfahrungen von Deprivation. Da uns keine Ressourcen für eine eigene Erhebung zur Verfügung standen, müssen wir uns an der bestehenden Datenlage orientieren. Eine Befragung rassistischer Einstellungen oder Abstiegserfahrungen ist uns daher nicht möglich. Erschwerend kommt hinzu, dass die statistischen Indikatoren in allen Städten erfasst sein müssen. Dies berück- 
sichtigend und im Anschluss an die oben eingeführten Debatten fokussieren wir auf vier Dimensionen:

Die Diskussionen, ob eher sozio-ökonomische oder identitätspolitische Faktoren ausschlaggebend sind, bilden einen zentralen Bezugspunkt. Wir fokussieren daher erstens auf die sozio-ökonomische Lage sowie zweitens auf die Präsenz von Migrant*innen im Viertel. Letzteres ist aus zwei Gründen unbefriedigend: Erstens fokussieren wir auf die Anwesenheit von Menschen, die potentiell von rassistischer Herabsetzung und Gewalt betroffen sind, anstelle Rassismus zu befragen; zweitens florieren rassistische Ressentiments auch bestens ohne den unmittelbaren Kontakt mit den vermeintlichen Anderen. Da das Thema Migration aber ein zentraler Mobilisierungsfaktor der AfD bei der Bundestagswahl 2017 war, befragen wir diese Dimension dennoch mit der gebotenen Vorsicht. Demokratieverdruss wurde oben als bedeutsamer Faktor für die Zuwendung zur extremen Rechten beschrieben, insofern befragen wir drittens den Zuspruch zur Demokratie. Hervorgehoben wurde in den Diskussionen auch die Relevanz der Altersstruktur, weshalb wir viertens auf diese fokussieren.

Zwecks Analyse müssen diese Dimensionen in konkrete statistische Indikatoren überführt werden. Wir betonen mit Manow (2018: 73), dass es sich bei Sozialdaten niemals um rein individuelle Verhältnisse, sondern um soziale handelt. Beispielsweise stehen hohe Arbeitslosenzahlen in einem Sozialraum nicht nur für viele individuell Betroffene, sondern sie prägen die soziale Umgebung mit und geben Aufschluss über das Milieu insgesamt. Dies gilt insbesondere, wenn es sich um so kleinräumige Einheiten wie Stadtteile handelt. So ist etwa auf Grund von Mietpreisen und Praxen der Exklusion unwahrscheinlich, dass in einem sehr reichen Viertel viele Menschen leben, die Arbeitslosengeld II beziehen. Für die Dimension sozio-ökomische Lage ziehen wir sodann zum einen den Anteil jener, die Arbeitslosengeld gemäß SGB II beziehen, und zum anderen den der sozialversicherungspflichtig Beschäftigten als Indikatoren heran. Dabei handelt es sich jeweils um den Anteil an der erwerbsfähigen Bevölkerung im Stadtteil (bzw. in Städten, in denen die Erwerbsfähigkeit nicht dokumentiert ist, der Bevölkerungsanteil von 15 bis 64 Jahren). Ersteres verstehen wir als Indiz für weniger wohlhabende Viertel, letzteres als Hinweis für eine verbreitete Wahrnehmung von Absicherung und Situiertheit. Für die zweite Dimension Migration greifen wir auf die beiden Indikatoren Anteil an Menschen mit Migrationshintergrund sowie an Ausländer*innen zurück. Die Daten für den ersten Indikator sind für die Städte Erfurt, Kiel, Rostock und Magdeburg nicht verfügbar und dort, wo sie vorliegen, unterschiedlich bestimmt. Zwecks Vereinheitlichung bestimmen wir Menschen mit Migrationshintergrund hier als Deutsche, die selbst oder deren Eltern eingewandert sind, und Personen ohne deutsche Staatsbürgerschaft. Als Indikator für das Empfinden von demokratischer In- bzw. Exklusion haben wir die Wahlbeteiligung herangezogen. Dabei interpretieren wir eine niedrige Beteiligung im Stadtteil als Indiz für eine stär- kere Verbreitung von Unzufriedenheit mit aktuellen demokratischen Prozessen. Um das Alter zu erfassen, haben wir den Alters- sowie Jugendquotienten in den Blick genommen. Ersterer wird in den Kommunen einheitlich als Menschen über 65 Jahre bestimmt, letzterer variiert, wobei es sich meist um Menschen unter $18^{1}$ handelt. Die Quotienten sind relative Werte, die den Anteil an Jugendlichen sowie an Alten in Relation zur erwerbsfähigen Bevölkerung setzen. Sie sind ein Indiz dafür, ob die mittleren Alter - also die vornehmlichen AfD-Wähler*innen - ihr Viertel eher als jung oder als alt wahrnehmen. Eine jeweils starke Ausprägung der Quotienten verdeutlicht aber auch absolut hohe Anteile junger bzw. alter Menschen.

Orte der Untersuchung sind die Stadtteile der jeweils größten Stadt je Bundesland im Jahr 2017 (vgl. Tabelle 1 im nächsten Kapitel). Für die Zusammenstellung des Datensatzes haben wir die statistischen Jahrbücher der Kommunen sowie deren Wahlanalysen herangezogen. In Kiel sind die räumlichen Einheiten der Erfassung der Sozialdaten nicht deckungsgleich mit den Wahlbezirken, weshalb eine Untersuchung nicht möglich war. Unser Datensatz birgt einige Schwächen: Erstens bestehen enorme Größenunterschiede zwischen den räumlichen Einheiten. Wir referenzieren deshalb auf die prozentualen Anteile je Stadtteil. Jedoch sind gerade in Berlin die Einheiten so viel größer als in den anderen Städten, dass ein Vergleich kaum sinnvoll und eine vertiefte qualitative Analyse schwierig ist. Zweitens werden dieselben Indikatoren nicht in allen Kommunen gleich erfasst und so weisen die Daten über die Städte hinweg, mit Ausnahme der Wahlbeteiligung, eine geringe Validität auf. Aus diesem Grund verzichten wir auf einen statistischen Vergleich zwischen den Städten sowie auf die Analyse multipler Regressionen. Wir analysieren die Zusammenhänge zwischen den ausgewählten Indikatoren (unabhängige Variablen) und ihrer Wirkung auf den Zweitstimmenanteil der AfD (abhängige Variable) je Stadtteil in den Städten jeweils für sich mittels einer bivariaten linearen Regression. In der Analyse lassen sich erste Tendenzen erkennen, die wir um eine relationale, qualitative Betrachtung der Einzelfälle ergänzen und mittels des Rückgriffs auf die Literatur vergleichend diskutieren. In allen Städten beobachten wir klare Diskontinuität im Gefüge der beschreibbaren Korrelationen. Wir betrachten diese genauer, zumal wir uns aus der Befragung des Spannungsverhältnisses Aufschlüsse über gesellschaftliche Entwicklungen erhoffen.

\section{Erfolgsbedingungen der AfD im städtischen Kontext}

An dieser Stelle widmen wir uns den empirischen Ergebnissen und setzen diese in Bezug zu den oben eingeführten Debatten. Wir werden nun zuerst die stadtteilbezogenen Wahlergebnisse der AfD in den unterschiedlichen Städten vor-

\footnotetext{
${ }^{1}$ Manchmal aber auch bis 15,16 bzw. 20 Jahre.
} 
Tabelle 1. AfD-Wahl je Stadt, inklusive Extremwerte und Standardabweichung sowie Anzahl der Stadtteile.

\begin{tabular}{|c|c|c|c|c|c|c|c|c|c|c|c|c|c|}
\hline $\begin{array}{l}\overrightarrow{\tilde{\sigma}} \\
\vec{\omega}\end{array}$ & 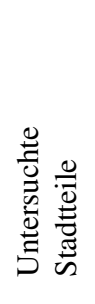 & 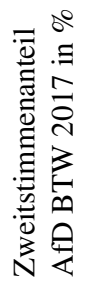 & 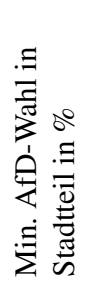 & 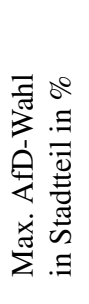 & 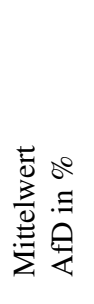 & 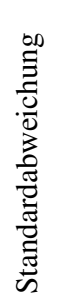 & $\frac{\overrightarrow{\tilde{J}}}{i}$ & 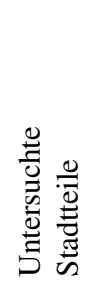 & 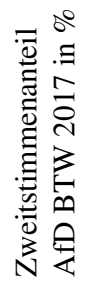 & 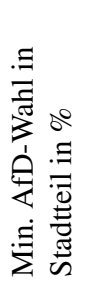 & 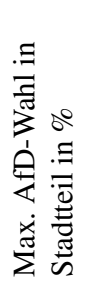 & 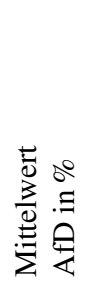 & 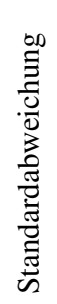 \\
\hline Berlin & 12 & 12,0 & 6,3 & 21,6 & 12,3 & 4,5 & Leipzig & 63 & 18,3 & 7,7 & 29,5 & 20,4 & 6,3 \\
\hline Bremen & 49 & 8,3 & 2,8 & 16,8 & 8,9 & 4,4 & Magdeburg & $29^{c}$ & 17,6 & 11,3 & 24,8 & 18,6 & 4,0 \\
\hline Erfurt & $44^{\mathrm{a}}$ & 18,5 & 14 & 33,3 & 23,1 & 4,3 & Mainz & 15 & 7,3 & 4,9 & 10,1 & 7,7 & 1,7 \\
\hline Frankfurt/M & $43^{b}$ & 8,6 & 4,5 & 14,5 & 9,5 & 2,7 & München & 25 & 8,4 & 4,5 & 13,2 & 8,4 & 2,3 \\
\hline Hamburg & 99 & 7,8 & 2,3 & 27,6 & 9,0 & 4,0 & Potsdam & $22^{d}$ & 12,8 & 9,3 & 22,2 & 14,2 & 3,9 \\
\hline Hannover & 49 & 8,3 & 3,7 & 18,2 & 9,2 & 3,4 & Rostock & $16^{\mathrm{e}}$ & 14,7 & 7,8 & 20,6 & 15,7 & 4,1 \\
\hline Kiel & 14 & 6,9 & 4,1 & 10,5 & 7,5 & 1,9 & Saarbrücken & 20 & 9,4 & 5,3 & 16,4 & 10,0 & 2,6 \\
\hline Köln & 86 & 7,4 & 3,1 & 17,5 & 9,1 & 3,4 & Stuttgart & 23 & 8,8 & 5,1 & 16,0 & 9,5 & 2,9 \\
\hline
\end{tabular}

War ein Indikator für einen Stadtteil nicht verfügbar, so wurde der Stadtteil, um einen einheitlichen Grundwert zu garantieren, aus dem Datensatz entfernt: ${ }^{a}$ Eigentlich 53 , aber Daten nicht vollständig; ${ }^{\mathrm{b}}$ Eigentlich 44, aber Daten nicht vollständig; ${ }^{\mathrm{c}}$ Eigentlich 32 , aber Daten nicht vollständig; ${ }^{\mathrm{d}}$ Eigentlich 29 , aber Daten nicht vollständig; ${ }^{\mathrm{e}}$ Eigentlich 21 , aber Daten nicht vollständig.

stellen, dann die Ergebnisse der linearen Regressionsanalyse beschreiben und diese anschließend diskutieren. Zumal die statistische Auswertung jeweils auf der Ebene der einzelnen Städte verbleibt, gilt: Wenn im Folgenden von ,hohen“ oder „geringen“ Anteilen des Stimmenanteils oder eines Indikators die Rede ist, dann bezieht sich dies immer auf die diskutierte Stadt und ist als relative und nicht absolute Beschreibung zu bewerten.

Innerhalb der einzelnen Städte sind die Wahlergebnisse zwischen den Stadtteilen, wie in Tabelle 1 deutlich zu sehen ist, stark polarisiert. In Hamburg und Leipzig beträgt die Differenz zwischen dem höchsten und dem tiefsten Wahlergebnis über $20 \%$; lediglich in Kiel, Mainz und München liegen sie in unserem Sample unter $10 \%$, überall sonst ist die Differenz zumindest zweistellig. Die oben bereits angesprochene Polarisierung zwischen Ost- und Westdeutschland wird deutlich sichtbar. Zum einen sind in den Städten Ostdeutschlands die Wahlergebnisse der AfD durchweg höher als in Westdeutschland: Erfurt (18,5\%), Leipzig (18,3\%), Magdeburg $(17,6 \%)$ und Rostock $(14,7 \%)$ sind die vier Städte mit dem höchsten Zuspruch in unserem Sample. Umgekehrt erzielte die Partei in keiner westdeutschen Stadt ein zweistelliges Resultat - am schlechtesten schnitt sie in Kiel (6,9\%) ab. Zum anderen fällt auf, dass in den Städten Ostdeutschlands in der Tendenz die Polarisierung der Wahlergebnisse (Standardabweichung) stärker ausfällt als im Westen. Besonders auffällig ist Leipzig mit einer Standardabweichung von 6,3. Der niedrigste Zuspruch zur AfD beträgt hier 7,7\% in der Südvorstadt, der höchste 29,5\% in Lausen-Grünau.

In Tabelle 2 sind die Ergebnisse der bivariaten linearen Regressionsanalyse zusammengefasst. Wichtig ist: Mittels der Regressionsanalyse können wir nur Aussagen darüber treffen, ob der Anteil an Menschen einer bestimmten Personen- gruppe innerhalb eines Stadtteils mit der Wahl der AfD korreliert; es ist keine Aussage darüber, dass die entsprechende Gruppe auch die Rechtsaußen-Partei wählt. Aus den aufgezeigten Korrelationen ist zudem nicht auf Kausalitäten zu schließen - sie geben Hinweise, sind aber kein Erklärendes. Die Werte des Regressionskoeffizienten sind getrennt voneinander und wie folgt zu interpretieren: Steigt die unabhängige Variable auf der Städteebene um einen Prozentpunkt, dann nimmt bei einem positiven Regressionskoeffizienten der Anteil der Stimmen für die AfD um den dargestellten Wert des Regressionskoeffizienten zu. Ein negativer Wert zeigt, dass die Regressionsgerade fällt und der Zuspruch beim Steigen der unabhängigen Variabel abnimmt. Die Erklärungsgüte des Modells wurde als signifikant interpretiert, wenn der $p$-Wert des F-Wertes niedriger als 0,05 ist und das adjustierte R-Quadrat höher als 0,1 liegt.

Die Ergebnisse sind heterogen, dennoch lassen sich relevante Zusammenhänge beschreiben. Am deutlichsten tritt der Indikator Wahlbeteiligung hervor. Mit Ausnahme von Erfurt, wo kein Zusammenhang sichtbar wurde, gilt: Je tiefer die Wahlbeteiligung, desto höher der Zweitstimmenanteil der AfD. In 8 Städten ist der Zusammenhang sehr signifikant ausgeprägt. In den Städten Berlin $\left(-0,687^{*}\right)$, Hamburg $\left(-0,347^{* * *}\right)$, Köln $\left(-0,339^{* * *}\right)$, Leipzig $\left(-0,367^{* *}\right)$ und Stuttgart $\left(-0,485^{* * *}\right)$ handelt es sich bei der Wahlbeteiligung um den über alle Werte hinweg am stärksten ausgeprägten Wert. In Bezug auf Menschen, die SGB II beziehen, beobachten wir in 11 von 15 Städten einen positiven $\mathrm{Zu}$ sammenhang mit dem Zweistimmenanteil der AfD. In 6 Fällen ist der Zusammenhang hoch signifikant. In den Städten München $\left(3,493^{* * *}\right)$, Frankfurt am Main $\left(1,485^{* * *}\right)$, Mainz $\left(0,824^{*}\right)$, Hannover $\left(0,733^{* * *}\right)$, Bremen $\left(0,636^{* * *}\right)$ und Magdeburg $\left(0,604^{*}\right)$ handelt es sich hierbei - bisweilen mit Ab- 
Tabelle 2. Tabelle der Regressionskoeffizienten zwischen den unabhängigen Variablen zu dem AfD-Wahlergebnis in den Stadtteilen je Stadt.

\begin{tabular}{|c|c|c|c|c|c|c|c|c|}
\hline $\begin{array}{l}\overrightarrow{\tilde{J}} \\
\text { i }\end{array}$ & 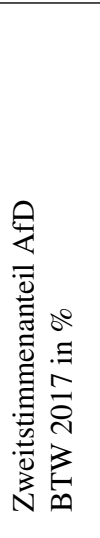 & 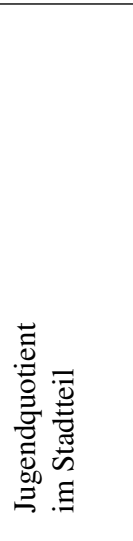 & 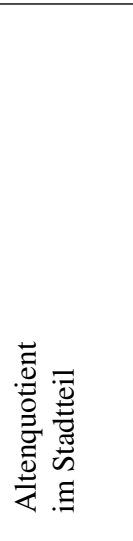 & 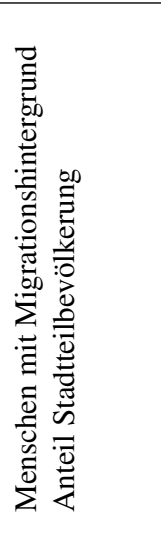 & 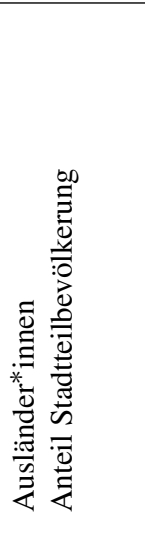 & 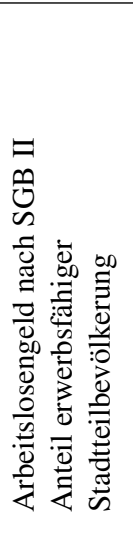 & 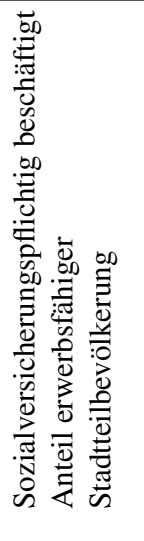 & 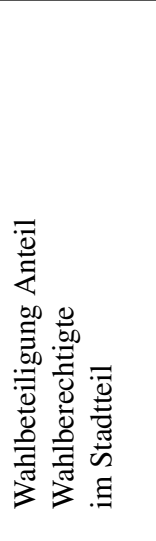 \\
\hline Berlin & 12,0 & 0 & 0 & $-0,280^{* *}$ & $-0,453^{* *}$ & 0 & 0 & $-0,687^{*}$ \\
\hline Bremen & 8,3 & $0,325^{* * *}$ & $0,143^{* *}$ & $0,232^{* * *}$ & $0,232^{* *}$ & $0,636^{* * *}$ & 0 & $-0,401^{* * *}$ \\
\hline Erfurt & 18,5 & 0 & $0,137^{*}$ & $\mathrm{x}$ & 0 & 0 & 0 & 0 \\
\hline Frankfurt/M & 8,6 & $0,203^{* *}$ & $0,165^{*}$ & $0,100^{* * *}$ & 0 & $1,485^{* * *}$ & $-0,391^{* *}$ & $-0,316^{* * *}$ \\
\hline Hamburg & 7,8 & $0,197^{* * *}$ & 0 & $0,136^{* * *}$ & $0,141^{* * *}$ & $0,221^{* * *}$ & 0 & $-0,347^{* * *}$ \\
\hline Hannover & 8,3 & $0,251^{* * *}$ & 0 & $0,184^{* * *}$ & $0,177^{* *}$ & $0,733^{* * *}$ & 0 & $-0,357^{* * *}$ \\
\hline Kiel & 6,9 & $\mathrm{x}$ & $\mathrm{x}$ & $\mathrm{x}$ & $\mathrm{x}$ & $\mathrm{x}$ & $\mathrm{x}$ & $-0,163^{*}$ \\
\hline Köln & 7,4 & $0,327^{* * *}$ & 0 & $0,169^{* * *}$ & $0,209^{* * *}$ & $0,223^{* * *}$ & 0 & $-0,339^{* * *}$ \\
\hline Leipzig & 18,3 & 0 & $0,237^{* * *}$ & $-0,325^{* * *}$ & $-0,345^{* *}$ & 0 & $0,308^{* *}$ & $-0,367^{* *}$ \\
\hline Magdeburg & 17,6 & $0,477^{*}$ & 0 & $\mathrm{x}$ & 0 & $0,604^{*}$ & 0 & $-0,290^{* *}$ \\
\hline Mainz & 7,3 & $0,204^{* *}$ & 0 & 0 & 0 & $0,824^{*}$ & 0 & $-0,247^{*}$ \\
\hline München & 8,4 & $0,485^{* * *}$ & $0,282^{* *}$ & $0,246^{* *}$ & $0,249^{*}$ & $3,493^{* * *}$ & 0 & $-0,520^{* * *}$ \\
\hline Potsdam & 12,8 & 0 & 0 & 0 & $0,446^{*}$ & $0,424^{* *}$ & 0 & $\mathrm{x}$ \\
\hline Rostock & 14,7 & $0,553^{*}$ & 0 & $\mathrm{x}$ & $0,828^{* *}$ & $0,761^{* *}$ & 0 & $-0,359^{* * *}$ \\
\hline Saarbrücken & 9,4 & $0,371^{* *}$ & 0 & 0 & 0 & $0,149^{* *}$ & 0 & $-0,315^{* * *}$ \\
\hline Stuttgart & 8,8 & $0,394^{* *}$ & 0 & $0,215^{* *}$ & 0 & 0 & 0 & $-0,485^{* * *}$ \\
\hline
\end{tabular}

$\mathrm{x}=$ Daten für diese Stadt nicht verfügbar.

$0=$ Regressionsmodell unzureichende Erklärungsgüte (zum F-Wert gehöriger $p$-Wert größer als 0,05 oder R-Quadrat kleiner als 0,1 ).

Signifikanzniveau: ${ }^{*} 0,01>p>0,5$ (signifikant), ${ }^{* *} 0,001>p>0,1$ (sehr signifikant), ${ }^{* * *} p=0,000$ (hoch signifikant).

stand - um die am stärksten ausgeprägten Regressionskoeffizienten. In 7 der 12 Städte, für die Daten zum Anteil an Menschen mit Migrationshintergrund vorliegen, ist der Zusammenhang positiv; in Leipzig $\left(-0,325^{* * *}\right)$ und Berlin $\left(-0,280^{* *}\right)$ hingegen negativ. Für die ostdeutschen Städte Erfurt, Magdeburg und Rostock liegen hier keine Daten vor. Der Anteil an Ausländer*innen ist besser erfasst. Wir beobachten in 7 von 15 Fällen eine positive Korrelation mit dem Zweitstimmenanteil der AfD; in Berlin $\left(-0,453^{* *}\right)$ und Leipzig $\left(-0,345^{* *}\right)$ ist auch hier der Zusammenhang negativ. In Erfurt, Frankfurt am Main, Magdeburg, Mainz, Saarbrücken und Stuttgart ergaben sich keine signifikanten Korrelationen. In Rostock $\left(0,828^{* *}\right)$ und Potsdam $\left(0,446^{*}\right)$ hingegen ist der Anteil an Ausländer*innen der über alle Werte hinweg am stärksten ausgeprägte Regressionskoeffizient. Wichtig scheint uns, dass insgesamt - mit Ausnahme der beiden genannten ostdeutschen Städte - die migrationsbezogenen Regressionskoeffizienten im Vergleich zum Anteil an SGB II und der Wahlbeteiligung deutlich niedriger ausfallen. Überrascht hat uns der Jugendquotient. In 11 von 15 Fällen korreliert dieser positiv mit der Wahl der AfD; in 5 Fällen ist die Ausprägung gar hoch signifikant. In Stuttgart $\left(0,394^{* *}\right)$, Saarbrücken $\left(0,371^{* *}\right)$ und Köln $\left(0,327^{* * *}\right)$ weist der Jugendquotient verglichen zu den anderen Variablen einen hohen Regressionskoeffizienten auf. Der höchste Wert findet sich im Städtevergleich in Rostock $\left(0,553^{*}\right)$. In Saarbrücken ist es gar der am stärksten ausgeprägte Regressionskoeffizient. Der Altersquotient hingen schlägt nur in 5 von 15 Fällen zu Buche und lediglich in Erfurt $\left(0,137^{*}\right)$, wo ansonsten keine Korrelationen auftreten, ist dieser an sich lediglich signifikante und schwach ausgeprägte Wert der am stärksten ausgeprägte. In den Städten, in denen beide altersabhängigen Quotienten Zusammenhänge aufweisen, besitzen jene für den Altenquotienten stets einen niedrigeren Regressionskoeffizienten.

\section{Multidimensionale Betrachtungen}

Für die weitere Diskussion der Ergebnisse folgen wir den vier Dimensionen sozio-ökonomische Lage, Migration, $\mathrm{Zu}-$ 
spruch zur Demokratie und Altersstruktur. Dabei binden wir diese an die oben eingeführten Debatten zurück und versuchen uns an einer multidimensionalen Betrachtung. Hier fokussieren wir auch auf die Diskontinuitäten innerhalb der Korrelationen, zumal wir uns aus der Betrachtung der Spannungsverhältnisse Rückschlüsse auf gesellschaftliche Tendenzen erwarten.

Wir beginnen mit der Diskussion der Dimension sozioökonomische Lage. Am deutlichsten ist der SGB-IIbezogene Zusammenhang in München, mit einem Regressionskoeffizienten von $3,493^{* * *}$. Dies ist der höchste Koeffizient in unserem Sample. Zurückzuführen sein dürfte diese Ausprägung auf die geringe Zahl an Bezieher*innen (max. 1,6\%) und eine gleichzeitig hohe Signifikanz der Korrelation. Hohe Werte des Regressionskoeffizienten im Verhältnis zu den anderen unabhängigen Variablen sind zudem in Frankfurt am Main $\left(1,485^{* * *}\right)$, Mainz $\left(0,824^{*}\right)$, Rostock $\left(0,761^{* *}\right)$, Hannover $\left(0,733^{* * *}\right)$, Bremen $\left(0,636^{* * *}\right)$ und Magdeburg $\left(0,604^{*}\right)$ zu beobachten. Die Ursachen für die relativ gesehen hohen Werte divergieren. In Rostock ist wesentlich, dass die Stadtteile mit dem höchsten Anteil an SGB-II-Bezieher*innen allesamt auch die sind, in denen die AfD sehr stark abschneidet - ansonsten sind die Verteilungen weniger linear; ähnliches gilt für Mainz, wenn auch auf einem deutlich tieferen Niveau der AfD-Wahl. In Frankfurt am Main fällt umgekehrt ins Gewicht, dass in den Stadtteilen mit den kleinsten Anteilen an SGB II die AfD eindeutig weniger Zuspruch erhält. Keine Korrelationen zwischen dem Anteil an SGB-II-Bezieher*innen und der Wahl der AfD können wir für Berlin, Erfurt, Leipzig und Stuttgart aufzeigen.

In Bezug auf den zweiten Indikator für die sozioökonomische Lage, den Anteil an sozialversicherungspflichtig Beschäftigten, gilt für unser Sample, dass lediglich in Leipzig und in Frankfurt am Main ein Zusammenhang mit dem Zweitstimmenanteil der AfD zu beobachten ist. Mit zunehmendem Anteil steigt in Leipzig die Zustimmung zur AfD, in Frankfurt am Main hingegen sinkt diese. In beiden Städten ist das Ergebnis zwar sehr signifikant, der Einfluss auf den Stimmenanteil ist jedoch in beiden Städten mit $-0,391^{* *}$ für Frankfurt am Main und 0,308** für Leipzig im Vergleich zu den anderen Werten mäßig. Mit diesen Ergebnissen können wir den Befund von Manow (2018: 91-95) nicht stützen, wonach gerade in Regionen mit einem hohen Anteil an sozialversicherungspflichtig Beschäftigten die AfD stark gewählt wird. Auch die Interpretation, dass es vormals noch gut integrierte Arbeiter*innen seien, die sich, von Abstiegsängsten geplagt, vermehrt gegen Zuwanderung wenden würden, lässt sich nicht bestätigen. Es scheint, dass im städtischen Kontext Absicherung auf dem Arbeitsmarkt meist auch mit einer einigermaßen gesicherten Position einhergeht und die von Manow beschriebenen Effekte von einem Gefühl gesellschaftlicher Situiertheit und Weltoffenheit zumindest wettgemacht werden (vgl. Blokland, 2011: 180). In der Tendenz untermauern unsere Befunde den Forschungsstand dahingehend, dass sozio-ökonomische Marginalisierung in
Westdeutschland ein stärkerer Prädiktor für die Wahl der AfD ist als in Ostdeutschland (vgl. Deppisch et al., 2019: $78 \mathrm{ff}$.). Zwar weist der Anteil an SGB-II-Bezieher*innen auch in Rostock $\left(0,761^{* *}\right)$, Magdeburg $\left(0,604^{*}\right)$ und Potsdam $\left(0,424^{* *}\right)$ zumindest signifikante Korrelationen mit dem Zweitstimmenanteil der AfD auf, mit Erfurt und Leipzig können wir aber auch in relevanten Metropolen Ostdeutschlands keine Korrelation nachweisen. Hinzu kommt, dass in Rostock $\left(0,828^{* *}\right)$ und Potsdam $\left(0,446^{*}\right)$ jeweils der Anteil an Migrant*innen stärker ins Gewicht fällt.

Die auf Migration bezogenen Indikatoren weisen in unserem Sample in vielen Fällen signifikante Korrelationen auf, fallen aber weniger eindeutig aus als die oben beschriebenen Indikatoren. Für einen Ost-West-Vergleich taugt insbesondere der Indikator Menschen mit Migrationshintergrund auf Grund der Lücken in der Erfassung nicht. Hier ist der Anteil Ausländer*innen aussagekräftiger, wobei ein eindeutiges Ost-West-Muster auch hier nicht auszumachen ist. Insgesamt wird deutlich, dass zur Interpretation ein detaillierterer Blick vonnöten ist. Drei Konstellationen sind auffällig und diese wollen wir genauer betrachten. Erstens, Rostock und Potsdam, hier ist es der Anteil an Ausländer*innen der über alle Werte hinweg jeweils die stärkste Ausprägung aufweist; zweitens, Berlin und Leipzig, wo beide migrationsbezogenen Indikatoren negative Zusammenhänge aufweisen; und drittens, Frankfurt am Main und Stuttgart, hier weist der Anteil an Menschen mit Migrationshintergrund einen zumindest sehr signifikant positiven Zusammenhang auf, während der Anteil Ausländer*innen sich als nicht signifikant erweist. Für den ersten Fall haben wir Rostock, den zweiten Leipzig und den dritten Frankfurt am Main genauer betrachtet.

In Rostock scheint das Bild auf den ersten Blick eindeutig. In keiner anderen Stadt ist der Zusammenhang zwischen starkem Abschneiden der AfD und einem hohen Anteil an Ausländer*innen im Viertel so stark ausgeprägt wie hier. Die bis heute wirkmächtigen Bilder des Pogroms von Lichtenhagen 1992 tun das ihre dazu, um ein vermeintlich eindeutiges Verständnis über die Verhältnisse in der Stadt zu bekommen (Mau, 2019: 221-225). Bei genauerer Betrachtung erweist sich die Situation jedoch als komplexer. Die beiden Stadtteile mit dem höchsten Anteil an Ausländer*innen sind die eher zentrumsnahen, aber von Einfamilienhaussiedlungen geprägten Viertel Biestow und Gartenstadt/Stadtweide. Hier schneidet die AfD allerdings unterdurchschnittlich ab. Umgekehrt ist der Wahlanteil der AfD in Dierkow-Ost und -West sehr hoch, während dort praktisch keine Ausländer*innen wohnen. Hier handelt es sich um zwei Siedlungen, die in der zweiten Hälfte der 1930er Jahre als Wohnraum für Arbeiter*innen entstanden. An die Siedlungen grenzt Dierkow-Neu, eine Großwohnsiedlung aus Zeiten der DDR. Hier leben wie in anderen Großwohnsiedlungen, beispielsweise Lütten und Groß Klein oder Lichtenhagen, überdurchschnittlich viele Ausländer*innen und der Wahlanteil der AfD ist sehr hoch. Für die starke Ausprägung des Koeffizienten ist gerade dieser Zusammenhang 
zentral. Studien belegen, so Steffen Mau (2019: 229), diese Spaltung der Stadt. Zwar sei Rostock relativ weltoffen, aber gerade die ,alteingesessenen und homogenen Gruppen“ seien nur ,schwer zu motivieren, sich auf Migranten einzulassen“. Räumlich manifestiere sich dies stark in der sozialräumlichen Polarisierung ,zwischen den innerstädtischen Quarteiern und den Neubaugebieten“. Der Zuzug von Menschen aus anderen Teilen der Welt würde gerade von Bewohner*innen der Großwohnsiedlungen, die ohnehin vielfach auf stark von Frakturen geprägte Biographien blicken würden (Mau, 2019: $13 \mathrm{ff}$.), als weitere Veränderung empfunden, die schlicht nicht gewollt sei (Mau, 2019: 230). Es gehe um Gefühle des sozialen und kulturellen Abstiegs, der sich mit rassistischen Ressentiments vermenge. Hinzu komme, dass die AfD diese Viertel auch gezielt als Interventionsfelder definiert habe und sie politisch bearbeite (Mau, 2019: 225). Einhellig Hochburgen der extremen Rechten sind sie aber nicht. So schneiden in den Großwohnsiedlungen Rostocks die SPD wie auch Die Linke ebenfalls sehr gut ab.

In Leipzig, dem zweiten Beispiel, ist die starke Polarisierung der Stadt augenscheinlich. Auf der einen Seite Stadtteile wie Lindenau, Neustadt-Neuschönefeld, Plagwitz, Volkmarsdorf oder die Zentrums-Bezirke, wo die AfD im städtischen Vergleich schlecht abschneidet. Hier ist der Anteil an Menschen mit Migrationshintergrund hoch bis sehr hoch, jener sozialversicherungspflichtig Beschäftigter eher tief und die Anteile an SGB-II-Bezieher*innen liegen im unteren Bereich. Auf der anderen Seite schneidet die AfD in Stadtteilen wie Althen-Kleinpösna, Holzhausen, Liebertwolkwitz oder Seehausen sehr stark ab, wo kaum Menschen mit Migrationshintergrund sowie Ausländer*innen wohnen und auch die Werte für den SGB-II Bezug sehr gering sind. Der Anteil sozialversicherungspflichtig Beschäftigter hingegen ist sehr hoch. Im ersten Fall handelt es sich um urbane Gründerzeitviertel, die historisch durch einen eher mangelhaften Wohnungsbestand, günstige Mieten und geringe Zugangsrestriktionen geprägt waren. Hier haben sich linke und pluralistische Milieus entwickelt und Migrant*innen ein Zuhause gefunden. Dies spiegelt sich auch in den hohen Stimmanteilen für Die Linke und die Grünen wider. Auf Grund des enormen Drucks auf die Gründerzeitviertel und deren bisweilen rasanten Aufwertung in den letzten Jahren ist es Menschen mit kleinen und mittleren Einkommen sowie Ausländer*innen meist sind es in Leipzig nach 2015 Geflüchtete, die zuziehen - kaum noch möglich, sich hier niederzulassen. Sie können sich einerseits die Wohnungen nicht leisten, andererseits sind sie auch in diesen Vierteln von rassistischen Exklusionspraxen auf dem Wohnungsmarkt betroffen (Hummel et al., 2017). Wohnraum finden sie fast ausschließlich in den Großwohnsiedlungen wie Paunsdorf oder Grünau, wo die AfD stark abgeschnitten hat - sie gehören bundesweit $\mathrm{zu}$ den AfD-Hochburgen (Zschocke, 2019) und dort sind auch die Anteile an Menschen mit Migrationshintergrund sowie Ausländer*innen mittlerweile hoch. Würden alleine die urbanisierten Stadtteile von Leipzig betrachtet, ist davon auszu- gehen, dass das Regressionsmodell zwischen diesen beiden Polen eine unzureichende Güte erlangt hätte. Dass dem nicht so ist und eine negative Korrelation zu beobachten ist, liegt am zweiten, eingangs beschriebenen Cluster. Hierbei handelt es sich im Wesentlichen um eingemeindete Vororte von Leipzig, die stark von Einfamilienhaussiedlungen und eher mittelständischen bis wohlhabenden sowie eher konservativen Milieus geprägt sind. Die negative Korrelation ist daher wesentlich aus einer spezifischen Geschichte der Urbanisierung, Exklusion und des Zuzugs sowie Eingemeindungen heraus zu erklären.

Auch im dritten Fall Frankfurt am Main sind Fragen von Gentrifizierung und Exklusion zentral, wenn auch auf andere Weise. Eine Erklärung, warum der Anteil an Menschen mit Migrationshintergrund einen höchst signifikanten Zusammenhang aufweist, der Anteil Ausländer*innen jedoch nicht, birgt die Betrachtung von abermals zwei Clustern: zum einen jene Viertel, in denen die AfD schwach abschneidet und der Anteil an Ausländer*innen hoch, der Anteil an Menschen mit Migrationshintergrund jedoch unterdurchschnittlich ist. Zum anderen sind es (ehemalige) Arbeiter*innenviertel, wo die AfD heute stark abschneidet, die teilweise seit Jahrzehnten durch internationale Zuwanderung geprägt sind. Hier ist der Anteil an Menschen mit Migrationsgeschichte hoch, der Anteil an Ausländer*innen jedoch unterdurchschnittlich. Im Vergleich zwischen den Städten sind die Werte mit um die $25 \%$ jedoch hoch. Im ersten Cluster sind es Viertel wie Westend-Nord, Bockenheim oder Ostend. Es sind im Falle des Westends sowie von Bockenheim zentrumsnahe Stadtteile, in denen in den letzten Jahr(zehnt)en erhebliche Gentrifizierungsprozesse stattgefunden haben, Sozialwohnungen sukzessive ohne Ersatz weggefallen und die Mieten enorm gestiegen sind (Schipper und Heeg, 2020). Menschen mit kleinerem und mittlerem Einkommen, darunter viele Familien der einstigen Gastarbeiter*innen, wurden schon im Nachgang an die Häuserkämpfe der 1970er Jahre sukzessive verdrängt. Etabliert haben sich eher wohlhabende Milieus, in denen die SPD und die CDU - immer stärker aber auch die Grünen sowie Die Linke - ihre Basis finden. Im Ostend ist die Situation vergleichbar mit der Entwicklung in der Leipziger Innenstadt, zumal hier die Gentrifizierungsprozesse erst jüngst mit der Fertigstellung des neuen Sitzes der Europäischen Zentralbank erheblich Fahrt aufgenommen haben (Mösgen und Schipper, 2017). In allen drei Vierteln sind es heute nicht zuletzt die transnationalen Professionals, die in den Banken, Anwaltskanzleien und im akademischen Betrieb arbeiten, die hier den (internationalen) Zuzug bestimmen. Im zweiten Cluster sind es Stadtteile wie Bonames, Praunheim, Riederwald oder Schwanheim. Die Zuwachsraten der Ausländer*innen sind seit 2012 überall hoch und die Ankommenden sind hier anders als in den gehobenen Wohnlagen eher Menschen mit kleinem Einkommen, vielfach auch Geflüchtete, und sie werden als weitere Belastung von ohnehin marginalisierten und peripherisierten Nachbarschaften betrachtet (Mullis, 2021: 137 ff.). In diesen Vierteln werden 
mitunter tradierte rassistische Denkmuster offenkundig, zumal dort in den späten 1980er und frühen 1990er Jahren bereits die Republikaner Erfolge zu verbuchen vermochten (Mullis, 2021: 135-137). Bedeutsam ist hier, dass viele, die statistisch als Menschen mit Migrationshintergrund erfasst werden, Deutsche und damit wahlberechtigt sind. Ressentiments gegen die neue Zuwanderung werden sodann auch von ehemaligen Gastarbeiter*innen hervorgebracht und gerade Spätaussiedler*innen sind immer wieder durch ihre starke Zustimmung für die AfD aufgefallen. Es ist also bei weitem nicht so, dass eine Migrationsgeschichte vor Rassismus und Affinität zur AfD schützen würde - dies müsste bei einer genaueren Analyse mitberücksichtigt werden.

Resümierend halten wir für die Dimension Migration fest, dass, selbst wenn eine Regression auftritt, diese mit Vorsicht zu bewerten ist. Unser Beitrag zur Kontroverse über die Konflikt- bzw. Kontakthypothese liegt darin, dass wir dafür plädieren, dass der Anteil an Menschen mit Migrationshintergrund oder Ausländer*innen immer nur als Proxy, als ein Einstieg in die konkreten Situationen vor Ort betrachtet werden sollte. Hinzu kommt, dass Verschränkungen mit der Dimension sozio-ökonomische Lage zu vermuten sind. Anhand der Beispiele Leipzig und Frankfurt am Main legen wir nahe, dass es auf die Betrachtung tradierter Rassismen und urbaner Exklusionsmechanismen sowie Konfliktlagen ankommt. Migration dürfte nur dann zu einem Prädiktor für die Wahlstärke der AfD werden, wenn sie vor Ort auch als Problem gedeutet wird. Insofern kommt es in hohem Maße auf die kleinteiligen sozialen, ökonomischen und politischen Verhältnisse an.

Hinweise auf die Funktionsweise der lokalen Mechanismen gibt es. Johannes Hillje (2018: 9) etwa beschreibt für AfD-Hochburgen in Städten, dass Migration dort sehr wohl als das „größte Problem“ benannt wird. Dabei störe vor allem, dass die (angeblich) hohe Zahl an Migrant*innen zu einer sozialpolitischen Benachteiligung deutscher Staatsbürger*innen führe. Es gehe also weniger um Zuwanderung an sich als um Verteilungskämpfe. In eine ähnliche Richtung argumentieren Peter Bescherer et al. (2019: $23 \mathrm{ff}$.), wenn sie herausarbeiten, dass seitens der extremen Rechten „sozial Benachteiligte gegeneinander“ ausgespielt werden. Das Resultat: Die ständige Betonung, dass Geflüchtete durch Steuergelder finanziert würden, sowie der Vorwurf, dass Geflüchtete am Wohnungsmarkt Vorteile hätten, „produzieren Sozialneid und das Gefühl einer Benachteiligung der Einheimischen“. Für East London verweist Justin Gest (2016: 73, 162) auf einen weiteren Aspekt. Zuwanderung werde in den einstmals weißen Vierteln der Arbeiter*innen weniger ökonomisch als Bedrohung wahrgenommen als hinsichtlich der sozialen Position: Mit prekären Lebensverhältnissen hätte man sich abgefunden, bisweilen gar einen Stolz als Arbeiter*in behalten, sich heute allerdings auf der gleichen Stufe wie die Migrant*innen wiederzufinden, wolle man nicht hinnehmen. So keime der Hass auf vermeintliche Ausländer*innen gerade deshalb, weil diese als Sinnbild für den eigenen Abstieg wahrgenommen wür- den. Es geht um das Gefühl des Verlustes der privilegierten Zugehörigkeit zu einem Ort, einer Stadt oder einem Staat.

Abschließend wollen wir nochmals auf die beiden Dimensionen Zuspruch zur Demokratie sowie Altersstruktur zu sprechen kommen. Bei der Wahlbeteiligung, die wir als Indikator für den Zuspruch zur Demokratie verwendet haben, handelt es sich um das einzige Set an Daten in unserem Sample, das über alle Städte hinweg eine hohe Validität aufweist. Für die Wahlbeteiligung können wir demnach auch einen expliziten Vergleich zwischen Ost- und Westdeutschland vornehmen. Wenn auch unsere Daten noch rudimentär sind, legen die Ergebnisse dennoch nahe, dass das Gefühl, aus demokratischen Prozessen exkludiert zu sein (Heitmeyer, 2018: 177-196; Mullis, 2021: 140-144), in der Ursachensuche für das Erstarken der extremen Rechten im städtischen Kontext stärker gewichtet werden muss. Kein anderer Indikator hat so durchgehend eine Korrelation zu Tage geführt, wie der Zusammenhang zwischen einer niedrigen Wahlbeteiligung und dem starken Abschneiden der AfD. Im Vergleich zwischen Ost- und Westdeutschland (Abb. 1) fällt neben den insgesamt höheren Zweitstimmenanteilen für die AfD im Osten des Landes auch die deutlich größere Spreizung der Wahlergebnisse in Relation zur Wahlbeteiligung auf. Während im Westen eine hohe Wahlbeteiligung ausnahmslos mit einem niedrigen Zweitstimmenanteil der AfD einhergeht, gilt dies für Ostdeutschland nicht. Wir beobachten also im städtischen Kontext ein Phänomen, das auch für die Landtagswahl in Thüringen 2019 beschrieben wurde (Richter et al., 2019: 12): Ein starkes Abschneiden der AfD mit gleichzeitig (sehr) hoher Wahlbeteiligung - wenn auch es im Osten Stadtteile gibt, wo die Wahlbeteiligung sehr hoch ist und die AfD kaum Zuspruch erhält. Zwei Faktoren dürften für das erstgenannte Phänomen verantwortlich zeichnen: Zum einen, dass die AfD mancherorts ihr Potential insbesondere aus Angehörigen der vermeintlichen bürgerlichen Mitte zieht, die in Stadtteilen leben, wo die Wahlbeteiligung immer schon höher war; zum anderen, dass die autoritäre Reintegration durch die AfD mancherorts vormalige Rückstände in der Wahlbeteiligung wettmacht.

Hinsichtlich der Altersstruktur erweist sich gerade der Jugendquotient als relevante Größe, dies zeigt sich vor allem in Rostock $\left(0,553^{*}\right)$. Die AfD schneidet dennoch tendenziell in Stadtteilen stärker $a b$, in denen der Anteil junger Menschen das öffentliche Bild prägt. Vor dem Hintergrund der bisherigen Forschung, die vor allem auf die Überalterung von Kommunen abhebt (Franz et al., 2018), haben uns die Ergebnisse überrascht. Für eine solide Bewertung fehlt uns die Grundlage. Gründe könnten etwa in der Demografie der Stadtteile liegen. So leben in stark von Einwanderung geprägten Vierteln oftmals mehr Kinder, zumal der Altersschnitt der Zugezogenen meist jünger ist als jener der Alteingesessenen (Blokland, 2011: 180-186). Deutlich wird auf jeden Fall, dass im urbanen Kontext auch hier ein wenig betrachteter Zusammenhang evident wird. In Bezug auf die Altersstruktur ist am ehesten noch eine deutliche Ost-West-Differenz auszu- 

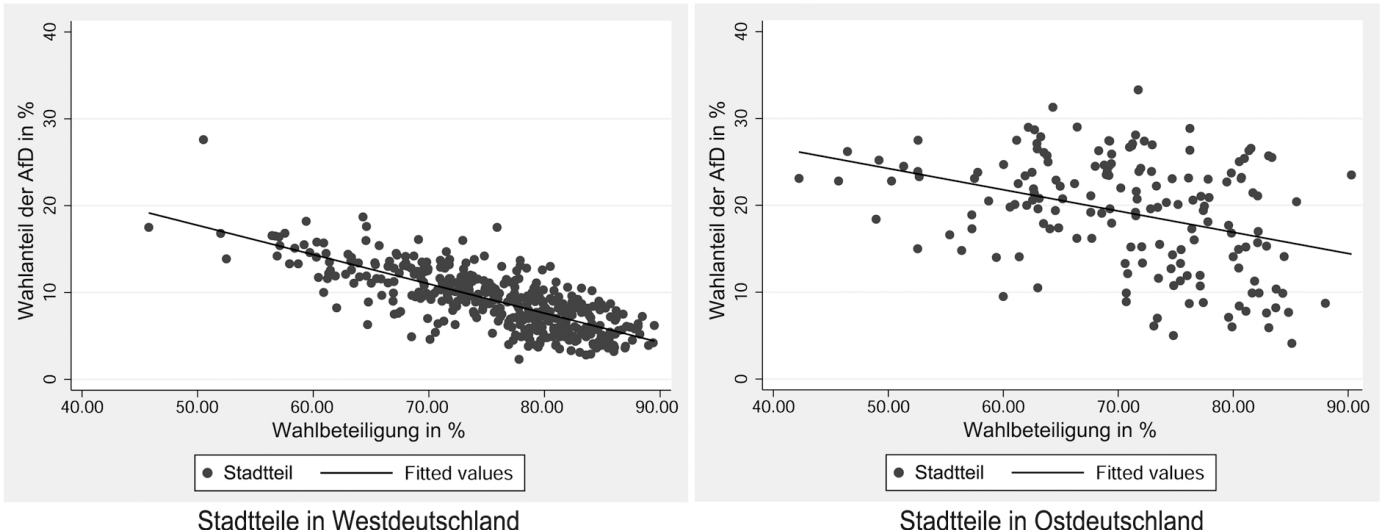

Stadtteile in Ostdeutschland

Abb. 1. Streudiagramm der AfD-Wahl in West- und Ostdeutschland (ohne Berlin) in Relation zur Wahlbeteiligung jeweils in Prozent je Stadtteil.

machen: So sind es die Städte Erfurt, Leipzig und Potsdam, in denen der Jugendquotient keine Rolle spielt, während in Erfurt $\left(0,137^{*}\right)$ und Leipzig $\left(0,237^{* * *}\right)$ der ansonsten weit weniger relevante Altersquotient einen signifikant positiven Zusammenhang aufweist.

In der getrennten Betrachtung der Indikatoren geht ein Ergebnis der Untersuchung unter: Insbesondere für Erfurt aber auch für Berlin, Magdeburg, Mainz, Saarbrücken und Stuttgart haben die gewählten Indikatoren kaum signifikante Korrelationen zu Tage geführt. In diesen Städten ist das Bild fragmentierter und schwieriger zu interpretieren. In Erfurt, wo der Stimmenanteil für die AfD insgesamt sehr hoch ist, liefert mit Ausnahme des Altenquotienten $\left(0,137^{*}\right)$ kein Indikator ein signifikantes Ergebnis. Zum Vergleich, Die Linke sowie SPD erzielen hier ihre stärksten Ergebnisse eindeutig in den Vierteln, in denen der Anteil der Menschen, die SGB II beziehen (SPD: 0,162 ${ }^{* * *} /$ Die Linke: $0,255^{* * *}$ ), sowie der Ausländer*innen hoch ist (SPD: 0,362***/Die Linke $0,600^{* * *}$ ). Wir schließen daraus, dass die AfD in Erfurt quer durch alle Milieus und Schichten starken Zuspruch erhält. In Magdeburg, Mainz und Saarbrücken sind es neben dem Jugendquotienten noch der Anteil an SGB-II-Bezieher*innen und die Wahlbeteiligung, die signifikante Zusammenhänge mit dem Stimmenanteil der AfD aufweisen. In Stuttgart ist es anstelle des Indikators SGB II der Anteil an Menschen mit Migrationshintergrund, in Potsdam anstelle des Jugendquotienten der Anteil an Ausländer*innen. Gerade in den genannten westdeutschen Städten schneidet die AfD jedoch insgesamt eher schlecht ab und die Standardabweichung ist klein, was uns einen ähnlichen Schluss wie für Erfurt ziehen lässt, aber auf deutlich tieferem Niveau der AfD-Ergebnisse. Auffällig ist zudem, dass unsere Indikatoren mit Ausnahme von Berlin, wo sehr großräumige Einheiten erfasst wurden, und Stuttgart gerade in kleineren Städten weit weniger signifikante Zusammenhänge mit dem Zweitstimmenanteil der AfD aufweisen, als dies in den größeren Städten der Fall ist.

\section{Fazit - Einige Antworten, Fragen bleiben}

Wir haben in diesem Beitrag nach der Wirkmächtigkeit der vier Dimensionen sozio-ökonomische Lage, Migration, $\mathrm{Zu}$ spruch zur Demokratie und Altersstruktur auf den Anteil der Zweitstimmen der AfD bei der Bundestagswahl 2017 in Städten gefragt. Mit unserer Analyse der Wahlergebnisse sowie von Sozialdaten auf der Ebene von Stadtteilen in der jeweils größten Stadt je Bundesland können wir vier Aspekte darlegen. Erstens, Städte sind hinsichtlich des Zuspruchs zur AfD erheblich und kleinteilig polarisiert; sie sind somit, zweitens, bei weitem nicht homogene, progressive Orte; drittens divergieren die Gründe der Polarisierung innerhalb der einzelnen Städte; und viertens ist in den Zusammenhängen keine klare Ost-West-Polarisierung auszumachen.

Grob können aus den untersuchten Städten zwei prototypische Stadtteile benannt werden, in denen die AfD stark abschneidet: Erstens, und dies ist das prägendste Muster in unserem Sample, Stadtteile, die sozio-ökonomisch marginalisiert, von einem hohen Anteil an Migrant*innen sowie von einem hohen Jugendquotienten und einer niedrigen Wahlbeteiligung geprägt sind. Statistisch finden wir diese eher in westdeutschen Städten als in Ostdeutschland, aber auch Rostock und Potsdam tendieren in dieser Richtung. Zudem haben wir in unserer vertieften Analyse auch in Leipzig Stadtteile gefunden, auf die diese Beschreibung zutrifft. Beim zweiten Typ Stadtteile handelt es sich um sozio-ökonomisch eher gehobene Viertel, die historisch konservativ geprägt sind, wo die Wahlbeteiligung hoch ist, kaum Arbeitslosigkeit herrscht und auch kaum Migrant*innen leben. Wir haben diese primär für Leipzig beschreiben können, finden solche Stadtteile aber auch in Frankfurt am Main und Potsdam. In Leipzig handelt es sich insbesondere um in jüngerer Zeit eingemeindete und/oder von Eigenheimsiedlungen geprägte Stadtteile. Ein drittes Phänomen können wir auf der Ebene von Städten in Ost und West beschreiben. Es sind Städte, in denen die Indikatoren kaum mit dem Stimmenanteil für 
die AfD korrelierten und davon auszugehen ist, dass in diesen Städten, auf hohem oder niedrigem Niveau, die AfD quer durch alle sozialen Milieus Zuspruch findet. In Bezug auf die leitende These schließen wir, dass die AfD sehr wohl in eher marginalisierten Stadtteilen stärker abschneidet als in besser gestellten, was aber weder heißt, dass sie dies in allen marginalisierten Stadtteilen tut, noch dass sie nicht auch in eher wohlhabenden Vierteln gut abschneiden kann.

Deutlich wird, dass die gesellschaftlichen Bruchlinien des Erstarkens der extremen Rechten nicht nur zwischen Ostund West sowie Stadt und Land, sondern eben auch mitten durch die Städte in Ost wie West verlaufen. Das für die Städte gezeichnete Bild ist ein weiterer Beleg dafür, dass es den Osten und den Westen so nicht gibt. Unterschiede sind nicht zu negieren, sie lassen sich aber keinesfalls auf binäre Territorialisierungen reduzieren. Mit Blick auf die Ursachensuche für das Erstarken der extremen Rechten in Deutschland ist uns Folgendes wichtig: Die polarisierte Debatte, ob nun eher sozio-ökonomische oder identitätspolitische Faktoren ausschlaggebend sind, ist nicht zielführend. Die Ergebnisse deuten darauf, dass keinem der Indikatoren ein Primat zugesprochen werden kann und sie in ihrer Multidimensionalität und Wechselwirkung zu betrachten sind. Gezeigt haben wir insgesamt, wie wichtig es ist, politische Prozesse auch in eher kleinen räumlichen Skalen zu betrachten. Dennoch, letztlich stoßen auch wir mit den statistischen Methoden an Grenzen. Die wichtige Frage nach Prozessen der Willensbildung, wie genau die verschiedenen Indikatoren wirkmächtig werden und ob - und wenn ja, wie - sie für die Menschen von Relevanz sind, lässt sich nur mittels qualitativer Forschung vor Ort herausarbeiten. Das bisweilen heterogene Bild unserer Erhebung zeigt: Es bedarf des lokal spezifischen Wissens, wenn die Muster entschlüsselt werden sollen - nur so kann das Bild schlüssiger und zugleich komplexer werden.

Datenverfügbarkeit. Der Datensatz ist zum Zeitpunkt der Veröffentlichung des Beitrags im Prozess der Veröffentlichung. Voraussichtlich im Juni 2021. Für Rückfragen kontaktieren Sie bitte Jan Lucas Geilen (geilen@students.uni-marburg.de).

Autorenmitwirkung. Das Vorhaben wurde von den Autoren gemeinsam entwickelt und umgesetzt. Entstanden ist es im Rahmen eines Praktikums an der HSFK im Projekt „Alltägliche politische Subjektivierung und das Erstarken regressiver Politiken“. Die statistischen Auswertungen sowie die Zusammenstellung des Datensatzes wurden von JLG vorgenommen.

Interessenkonflikt. The authors declare that they have no conflict of interest.
Danksagung. Wir bedanken uns bei den anonymen Gutachter*innen für die aufmerksame Lektüre und die konstruktive Kritik.

Begutachtung. This paper was edited by Nadine Marquardt and reviewed by three anonymous referees.

\section{Literatur}

Belina, B.: Zur Geographie der Abstiegsgesellschaft, Prokla, 47, 97-104, https://doi.org/10.32387/prokla.v47i186.181, 2017.

Belina, B.: Stadt, Land, AfD, Vortrag, Stadtallendorf, Deutschland, 21. November 2019.

Belina, B.: Political geography lecture: Social forms, spatial forms, and the New Right, Polit. Geogr., 81, 102091, https://doi.org/10.1016/j.polgeo.2019.102091, 2020.

Bernet, T., Bescherer, P., Beurskens, K., Feustel, R., und Michel, B.: Stadt von Rechts? sub $\backslash$ urban, 7, 7-22, https://doi.org/10.36900/suburban.v7i1/2.460, 2019.

Bescherer, P., Burkhardt, A., Feustel, R., Mackenroth, G., und Sievi, L.: Antiurbane Utopien, Podesta Working Paper 2, online aufrufbar: http://podesta-projekt.de/wp-content/uploads/2019/ 06/2019_06_21_WP2-Antiurbane-Utopien-webversion.pdf (letzter Zugriff: 8 April 2021), 2019.

Bhambra, G. K.: Brexit, Trump, and "methodological whiteness", The British Journal of Sociology, 68, 214-232, https://doi.org/10.1111/1468-4446.12317, 2017.

Blokland, T.: "Even when I see the real scoundrel around here, I don't feel unsafe", in: Die Besonderheit des Städtischen, Herausgeber: Herrmann, H., Keller, C., Neef, R., und Ruhne, R., VS, Wiesbaden, 173-196, 2011.

Brenner, N. und Theodore, N. (Hrsg.): Spaces of neoliberalism, Blackwell, Malden, ISBN 978-1-405-10105-9, 2002.

Bürk, T.: Gefahrenzone, Angstraum, Feindesland?, Westfälisches Dampfboot, Münster, ISBN 978-3-89691-894-9, 2012.

Celik, K., Decker, O., und Brähler, E.: Rechtsextremismus für die breite Gesellschaft?, in: Autoritäre Dynamiken, Herausgeber: Decker, O. und Brähler E., Psychosozial-Verlag, Gießen, 149175,2020

Decker, O., Kiess, J., Schuler, J., Handke, B., Pickel, G., und Brähler, E.: Methode, Ergebnisse und Langzeitverlauf, in: Autoritäre Dynamiken, Herausgeber: Decker, O. und Brähler, E., Psychosozial-Verlag, Gießen, 27-88, 2020.

Deppisch, L., Klärner, A., und Osigus, T.: Ist die AfD in ländlichen Räumen besonders erfolgreich?, in: Wissenschaft Demokratie, IDZ, 5, 75-87, https://doi.org/10.19222/201905/12, 2019.

Dowling, E., van Dyk, S., und Graefe, S.: Rückkehr des Hauptwiderspruchs?, Prokla, 47, 411-420, https://doi.org/10.32387/prokla.v47i188.69, 2017.

Förtner, M., Belina, B., und Naumann, M.: Stadt, Land, AfD, sub $\backslash$ urban, 7, 23-44, https://doi.org/10.36900/suburban.v7i1/2.483, 2019.

Franz, C., Fratzscher, M., und Kritikos, A.: AfD in dünn besiedelten Räumen mit Überalterungsproblemen stärker, DIW Wochenbericht, 8, 136-144, https://doi.org/10.18723/diw_wb:2018-8-3, 2018. 
Geiselberger, H. (Hrsg.): Die große Regression, Suhrkamp, Berlin, ISBN 978-3-518-07291-2, 2017.

Gest, J.: The new minority, Oxford Univ. Press, New York, ISBN 978-0-190-63255-7, 2016.

Gundelach, B.: Soziales Vertrauen in ethnisch heterogenen Nachbarschaften, SozProb, 28, 207-222, https://doi.org/10.1007/s41059-017-0034-7, 2017.

Heitmeyer, W.: Autoritäre Versuchungen, Suhrkamp, Berlin, ISBN 978-3-518-12717-9, 2018.

Hillje, J.: Rückkehr zu den politisch Verlassenen, Das Progressive Zentrum e.V, Berlin, online aufrufbar: https://www.progressives-zentrum.org/die-verlassenen/ (letzter Zugriff: 8 April 2021), 2018.

Hilmer, R., Kohlrausch, B., Müller-Hilmer, R., und Gagné, J.: Einstellung und soziale Lebenslage, Hans-Böckler-Stiftung, Workingpaper, ISSN 2509-2359, 2017.

Hövel, P.: Völkischer Nationalismus, Berichte, 92, 157-171, 2018.

Hummel, S., Krasowski, B., Midelia, S., und Wetendorf, J.: Rassistische Diskriminierung auf dem sächsischen Wohnungsmarkt, Antidiskriminierungsbüro Sachsen e.V., Leipzig, 2017.

Inglehart, R. und Norris, P.: Trump, Brexit, and the Rise of Populism, HKS Working Paper, RWP16-026, 2016.

Kaschuba, W.: Die Rache der Dörfer, Deutschlandfunk Kultur, 14. November 2016

Kipfer, S. und Dikeç, M.: Peripheries against peripheries? in: Massive Suburbanization: (Re-) Building the Global Periphery, Herausgeber: Güney, M., Keil, R., und Üçoğlu, M., University of Toronto Press, Toronto, 35-55, 2019.

Klüter, H.: Eine Krise regionaler Identität und ihr Gebrauchswert für rechtsgerichtete politische Gruppen - ein Beispiel aus Vorpommern, Geogr. Helv., 75, 151-167, https://doi.org/10.5194/gh-75-151-2020, 2020.

Lengfeld, H.: Der „Kleine Mann“ und die AfD, KZfSS, 70, 295310, https://doi.org/10.1007/s11577-018-0536-8, 2018.

Lizotte, C. A.: Where are the people?, Polit. Geogr., 71, 139-141, https://doi.org/10.1016/j.polgeo.2018.12.007, 2018
Manow, P.: Die Politische Ökonomie des Populismus, Suhrkamp, Berlin, ISBN 978-3-518-12728-5, 2018.

Mau, S.: Lütten Klein, Suhrkamp, Berlin, ISBN 978-3-518-428948, 2019.

Mösgen, A. und Schipper, S.: Gentrifizierungsprozesse im Frankfurter Ostend, Raumforschung und Raumordnung, 75, 125-141, https://doi.org/10.1007/s13147-016-0437-0, 2017.

Mullis, D.: Urban conditions for the rise of the far right in the global city of Frankfurt, Urban Stud., 58, 131-147, https://doi.org/10.1177/0042098019878395, 2021.

Mullis, D. und Zschocke, P.: Regressive Politiken und der Aufstieg der AfD, PRIF Report 5/2019, ISBN 978-3-946459-47-7, 2019.

Nachtwey, O.: Die Abstiegsgesellschaft, Suhrkamp, Berlin, ISBN 978-3-518-12682-0, 2016.

Oßenbrügge, J.: „March for Sozialgeographie“? Rechtspopulismus als Zumutung und die regressive Moderne als Herausforderung der Humangeographie, Geogr. Helv., 73, 309-319, https://doi.org/10.5194/gh-73-309-2018, 2018.

Quent, M.: Sonderfall Ost - Normalfall West? in: Rechtsextremismus und „Nationalsozialistischer Untergrund“, Herausgeber: Frindte, W., Geschke, D., Haußecker, N., und Schmidtke, F., Springer, Wiesbaden, 99-118, 2016.

Quent, M.: Deutschland rechts außen, Piper, München, EAN 9783-492-06170-4, 2019.

Richter, C., Salheiser, A., und Quent, M.: Rechtsradikale Landnahme, IDZ, Jena, online aufrufbar: https: //www.idz-jena.de/fileadmin/user_upload/IDZ_Analyse_ AfD-Wahlerfolg_Landtagswahlen_2019_FINAL_WEB.pdf (letzter Zugriff: 8 April 2021), 2019.

Schipper, S. und Heeg, S.: Wohnungspolitik in Frankfurt am Main, in: Lokale Wohnungspolitik, Rink, D. und Egner, B. (Hrsg.): Beispiele aus deutschen Städten, Nomos, Baden-Baden, 119-135, 2020.

Zschocke, P.: Im Schatten der Stadt, in: Leipziger Zustände 2018, Engagierte Wissenschaften e.V. (Hrsg.), 68-71, 2019. 